\title{
Evidence for long-range interactions between rare-earth impurity ions in nanocrystals embedded \\ in amorphous matrices with the two-level systems of the matrix
}

Copyright by the American Physical Society. Meltzer,

R. S. et al., "Evidence for

long-range interactions

between rare-earth impurity

ions in nanocrystals

embedded in amorphous

matrices with the two-level

systems of the matrix,"

Phys. Rev. B 64, 100201 (R)

DOI: http://

dx.doi.org/10.1103/

\author{
R. S. Meltzer, W. M. Yen, and Hairong Zheng \\ Department of Physics and Astronomy, The University of Georgia, Athens, Georgia 30602 \\ S. P. Feofilov \\ A. F. Ioffe Physical-Technical Institute, 194021 St. Petersburg, Russia \\ M. J. Dejneka \\ Corning Inc., Corning, New York 14831 \\ B. M. Tissue and H. B. Yuan \\ Department of Chemistry, Virginia Polytechnic Institute and State University, Blacksburg, Virginia 24061
}

(Received 19 March 2001; published 22 August 2001)

The low-temperature homogeneous broadening of the electronic transitions of $\mathrm{Eu}^{3+}$ and $\mathrm{Pr}^{3+}$ rare-earth impurity ions in $\mathrm{Y}_{2} \mathrm{O}_{3}$ and $\mathrm{LaF}_{3}$ nanocrystals embedded into amorphous materials (polymer and oxyfluoride glass ceramics) was studied with hole-burning and fluorescence line narrowing techniques. It is shown that the homogeneous linewidth is determined by the interaction of the impurity ions contained in the nanocrystals with the two-level systems (TLS's) of the surrounding glass matrix. A comparison of the experiments with a calculation provides direct evidence for the long-range nature of the interaction with the TLS's.

DOI: 10.1103/PhysRevB.64.100201

PACS number(s): 78.67.-n, 78.67.Bf
It is well known that when rare-earth (RE) ions are doped into glasses, their dynamics are governed by interactions with the two-level systems (TLS's) of the glass. Experiments in many systems provide evidence that at low temperatures the homogeneous linewidths, $\gamma_{h}$, in these systems obey a power law in temperature, $\gamma_{h} \sim T^{\alpha}$ with $1<\alpha<2$. Theoretical calculations show that this behavior is predicted for interactions with the TLS's of the glass. ${ }^{1-5}$ With the recent availability of nanocrystals containing RE ions, it is of great interest to determine whether RE ions separated from the glassy TLS's by the crystalline nanoparticle in which they are contained also exhibit interactions with TLS's when the nanoparticles are embedded in an amorphous matrix such as a glass. This can provide an independent test of the TLS model and can determine the length scale of the interactions.

It has been recently determined that materials consisting of insulating nanocrystals doped with RE ions embedded into amorphous (glassy) matrices possess nearly identical spectra to those of RE ions in single crystals of the same crystalline composition and structure. ${ }^{6}$ This is not unexpected, as the optical spectra of RE ions are determined by the short-range local environment of the RE site, which (with the exception of the ions at the nanocrystal-glass interface) remains unperturbed in crystallites of a few nanometers size. The sharp line spectra allow one to spectrally isolate ions in the nanoparticles from those in the amorphous matrix. Here we apply the technique of spectral hole burning to examine their dynamical properties. This work is motivated, in part, by the interest in these materials for applications such as hole-burning memories ${ }^{7}$ and optical processors. ${ }^{8,9}$

The mechanisms responsible for changes in the dynamical properties of the excited states of RE ions in insulating nanocrystals embedded in amorphous matrix, compared to single crystals, can be grouped into two categories: (i) those connected with size restriction effects and (ii) those caused by the interaction of RE ions with the amorphous environment surrounding the crystallites. The effects of the first kind are due mainly to the modification of the phonon spectrum of the nanocrystals at low frequencies due to their sizerestricted nature as reported for "free-standing" nanocrystalline materials produced by a variety of techniques. ${ }^{10-12}$ The effects of the second kind require mechanisms whose effective length scale is larger than the crystallite size. The interaction of RE ions in nanocrystals with excitations of the surrounding glass matrix are an example of the second and are the object of this investigation.

High-resolution spectral hole burning (HB) measurements as a function of temperature and particle size are performed in two different nanoparticle systems. The results are consistent with a recently reported study by Macfarlane and Dejneka ${ }^{13}$ on Tm-doped $\mathrm{LaF}_{3}$ nanoparticles in oxyfluoride glass. Here, however, we compare our measurements to the calculation of the interaction of RE ion with the TLS's where we can exclude the nanocrystal volume containing the RE ions. The comparison provides strong evidence for the dominance of interaction with the TLS's and enables the determination of the critical length scale of the long-range interaction.

The two different kinds of samples that were used in these experiments are (i) monoclinic $23 \mathrm{~nm} \mathrm{Y}_{2} \mathrm{O}_{3}: 0.1 \% \mathrm{Eu}^{3+}$ nanocrystals, produced by condensation after laser evaporation, ${ }^{14}$ which were dispersed in a siloxane polymer and (ii) transparent oxyfluoride glass ceramics ${ }^{6}$ consisting of a glassy matrix, in which $10-23 \mathrm{~nm} \mathrm{Eu}^{3+}$ or $\operatorname{Pr}^{3+}$ doped $\mathrm{LaF}_{3}$ crystallites are embedded.

The sharp line optical spectra of the $\mathrm{Y}_{2} \mathrm{O}_{3}: \mathrm{Eu}^{3+}$ nanocrystals embedded in the polymer ${ }^{14}$ did not show any significant changes relative to those of bulk powder samples and of free, 
as-prepared, nanoparticles of the same structure. The sharpline spectra of oxyfluoride glass ceramics containing $\mathrm{LaF}_{3}$ nanocrystals doped with $\mathrm{Eu}^{3+}$ and $\mathrm{Pr}^{3+}$ were also almost identical to those of $\mathrm{LaF}_{3}: \mathrm{Pr}^{3+}$ and $\mathrm{LaF}_{3}: \mathrm{Eu}^{3+}$ bulk single crystals. The main differences were some additional inhomogeneous broadening of the spectral lines $\left(\sim 1 \mathrm{~cm}^{-1}\right)$ and the existence of a broad fluorescence background resulting from the presence of some RE ions directly in the glassy component of the samples. ${ }^{15}$ The size of the nanocrystals in the glass ceramics could be controlled by annealing the samples at different temperatures. Electron and atomic force microscopy and x-ray diffraction were used for measurements of the size of the nanocrystals. A measurement of the average crystallite size is repeatable to $\pm 1.0 \mathrm{~nm}$ and $90 \%$ of the particles are within $7.5 \mathrm{~nm}$ of the average. The average RE ion concentration in the oxyfluoride ceramic samples was typically $0.005-0.05 \%$, but the RE concentration in the nanocrystals is usually a few times higher due to RE segregation. However, some of the RE ions are always present in the glass component.

Both of these materials consist of insulating nanocrystals of similar sizes $(\sim 10-25 \mathrm{~nm})$ embedded in an amorphous matrix. We interpret their general properties in terms of the same mechanism that we believe applies to both despite their independent preparation techniques and the difference in the amorphous matrix. This is supported by the fact that the dynamics of ions and molecules in amorphous media seem to show a universal behavior for a wide range of glassy media. $^{15,16}$

The samples were mounted in a liquid helium cryostat. For hole-burning experiments a few $\mathrm{mW}$ of $\mathrm{cw}$ light from a dye laser, Coherent CR599 (frequency jitter $\sim 4 \mathrm{MHz}$ ), was tuned to the ${ }^{7} F_{0^{-}}{ }^{5} D_{0}$ transition of $\mathrm{Eu}^{3+}$ or the ${ }^{3} H_{4}{ }^{-1} D_{2}$ transition of $\operatorname{Pr}^{3+}$ and loosely $(d \sim 0.5 \mathrm{~mm})$ focused into the sample. The transient hole spectrum [lifetime seconds (Pr) to minutes $(\mathrm{Eu})]$ was obtained with a repetitive burn/scan sequence (scan time $\sim 0.5 \mathrm{~s}$ ) using fluorescence detection with a photomultiplier tube through appropriate interference filters or a spectrometer. The data were averaged and stored on a digital oscilloscope. The hole widths obtained on single crystals, for which the homogeneous linewidth is much less than the spectral resolution for the HB technique, were used to estimate the instrumental contribution to the linewidth that was subtracted from all data.

We first describe the temperature dependence of the width of spectral holes, $\gamma_{\mathrm{HB}}$, burned in the ${ }^{7} F_{0^{-}}{ }^{5} D_{0}$ transition of $\mathrm{Eu}^{3+}$ ions located on the $c$ sites $(\lambda=582.8 \mathrm{~nm})$ in $23 \mathrm{~nm}$ $\mathrm{Y}_{2} \mathrm{O}_{3}$ monoclinic crystallites ${ }^{14}$ dispersed in siloxane polymer. These results are compared with the analogous HB data obtained with the free, as-prepared, nanocrystals of the same size. ${ }^{11}$ The results are shown in Fig. 1. The hole burning occurs due to redistribution of the population of the groundstate hyperfine levels of those ions whose hyperfine transitions are resonant with the laser. ${ }^{11,15}$ The sample with nanocrystals embedded into the polymer matrix exhibited a drastic increase in hole width and a very different power-law behavior for the temperature dependence of the hole widths relative to those of the same isolated nanoparticles. The $\sim T^{3}$ temperature dependence of the hole width observed for iso-

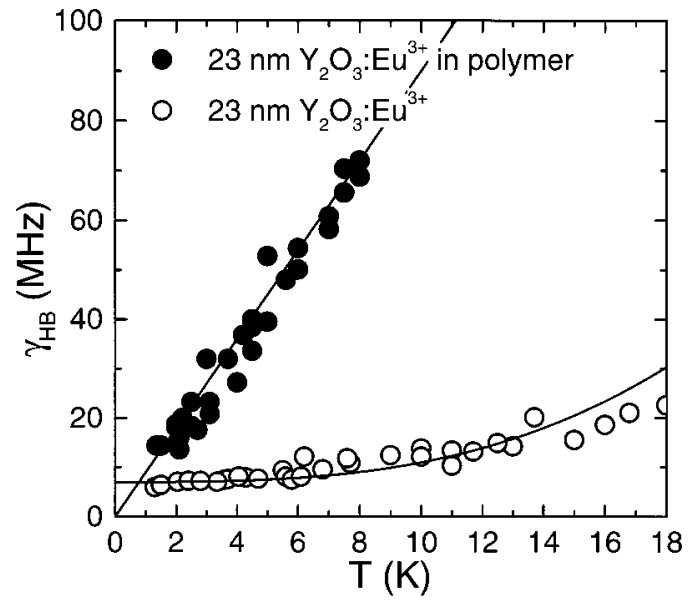

FIG. 1. Temperature dependence of the hole width for $\mathrm{Eu}^{3+}$ in $\mathrm{Y}_{2} \mathrm{O}_{3}$ nanocrystals. Solid lines: $\gamma_{\mathrm{HE}} \sim T$ (embedded in polymer) and $\gamma_{\mathrm{HB}} \sim T^{3}$ (isolated, as prepared).

lated nanoparticles ${ }^{11,12}$ becomes $\sim T$ for nanoparticles embedded in the polymer. Although the $\sim T^{3}$ behavior is not clearly evident for the $23 \mathrm{~nm}$ sample studied here, it is much more evident in the smaller particle samples which were part of the earlier study. ${ }^{11,12}$ The nearly linear temperature behavior of $\gamma_{\mathrm{HB}}$ in nanoparticles surrounded by the polymer matrix is very similar to that observed for $\mathrm{Eu}^{3+}$ doped glasses. ${ }^{15}$

The hole-burning results, which reflect the homogeneous linewidth $\gamma_{h}(T)$ of electronic transitions $\left(\gamma_{h}=\gamma_{\mathrm{HB}} / 2\right)$ suggest that the dominant mechanism for the homogeneous broadening of the ${ }^{7} F_{0^{-}}{ }^{5} D_{0}$ transition of $\mathrm{Eu}^{3+}$ in $\mathrm{Y}_{2} \mathrm{O}_{3}$ nanocrystals embedded in the polymer is identical to the TLS-RE ion interaction, which is responsible for homogeneous broadening found for ions in glasses. ${ }^{1-5,15,16}$ This interaction is usually assumed to be of an elastic dipole-dipole nature $^{2-5}$ and is thus relatively long range. We consider the possibility that such a long-range interaction may be effective at distances as large as the size of these nanoparticles and hence may be responsible for the homogeneous broadening of the optical transitions of impurity ions contained inside the nanocrystals.

In order to examine whether this temperature-dependent broadening is in fact a general property of RE-doped nanocrystals in amorphous media, oxyfluoride glass ceramic samples containing $\mathrm{LaF}_{3}$ nanocrystals $(0.05 \% \mathrm{Eu})$ were also studied. The hole burning again occurs due to the hyperfine mechanism. In Fig. 2 the temperature dependencies of widths of holes burned in the ${ }^{7} F_{0^{-}}{ }^{5} D_{0}$ transition of $\mathrm{Eu}^{3+}$ $(\lambda=578.2 \mathrm{~nm})$ are shown for a series of samples containing different particle size distributions. The nanocrystal size was varied by annealing the samples at different temperatures. ${ }^{6}$ The temperature dependence of the hole width for $\mathrm{Eu}^{3+}$ ions contained directly in the glassy matrix is also shown. The nearly linear temperature dependence of the hole width, $\gamma_{\mathrm{HB}} \sim T$, as well as the spectral dependence of the hole width as the laser is tuned inside the broad ${ }^{7} F_{0^{-}}{ }^{5} D_{0}$ transition of the glassy component (not shown) is very similar to that observed for other $\mathrm{Eu}^{3+}$ doped glasses. ${ }^{15}$ The character of the temperature dependencies of the hole widths for $\mathrm{Eu}^{3+}$ 


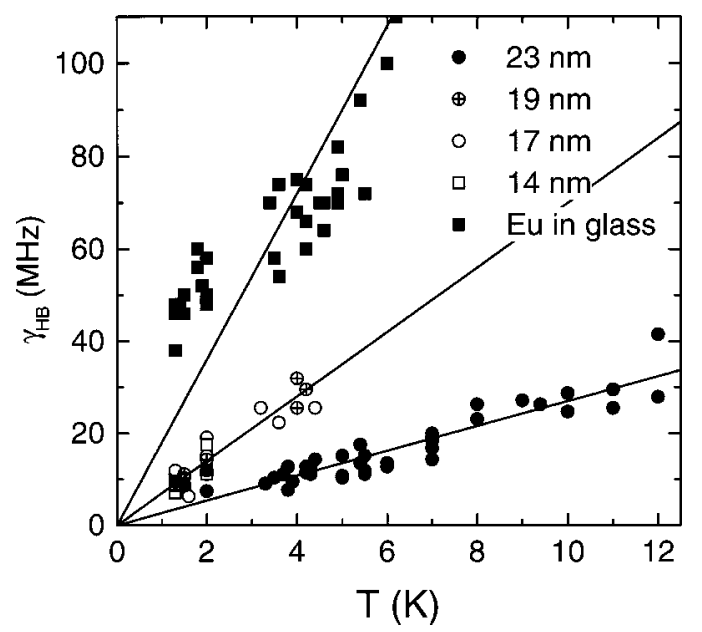

FIG. 2. Temperature dependence of the hole width for $\mathrm{Eu}^{3+}$ in $\mathrm{LaF}_{3}$ nanocrystals of different size embedded in oxyfluoride glass ceramics and for $\mathrm{Eu}^{3+}$ directly doped into the glass matrix. Solid lines: $\gamma_{\mathrm{HB}} \sim T$.

ions in nanocrystals embedded in the glass and for those ions contained directly in the glass matrix is similar $\left(\gamma_{\mathrm{HB}} \sim T\right)$, but the linewidth for the latter is significantly larger. It is important to note that the broadening decreases with an increase of nanocrystal size.

A nearly linear temperature dependence of hole width was also observed for holes burned on the ${ }^{3} \mathrm{H}_{4}{ }^{-}{ }^{1} \mathrm{D}_{2}$ transition of $\operatorname{Pr}^{3+}(\lambda=592.5 \mathrm{~nm})$ in oxyfluoride glass ceramics samples both for $\mathrm{Pr}^{3+}$ ions contained within the nanocrystals and for those located in the glass matrix-Fig. 3. Here hole burning at higher temperatures is due to photophysical processes involving rearrangement of the ions' surroundings. Such behavior, similar to that observed for $\mathrm{Pr}^{3+}$ ions in glasses, where it was found ${ }^{15}$ that $\gamma_{\mathrm{HB}} \sim T^{1.3}$, further supports the idea that interactions of the ions contained in the nanocrystals with the TLS's of the glass dominate the optical dephasing.

The results of these hole-burning measurements suggest

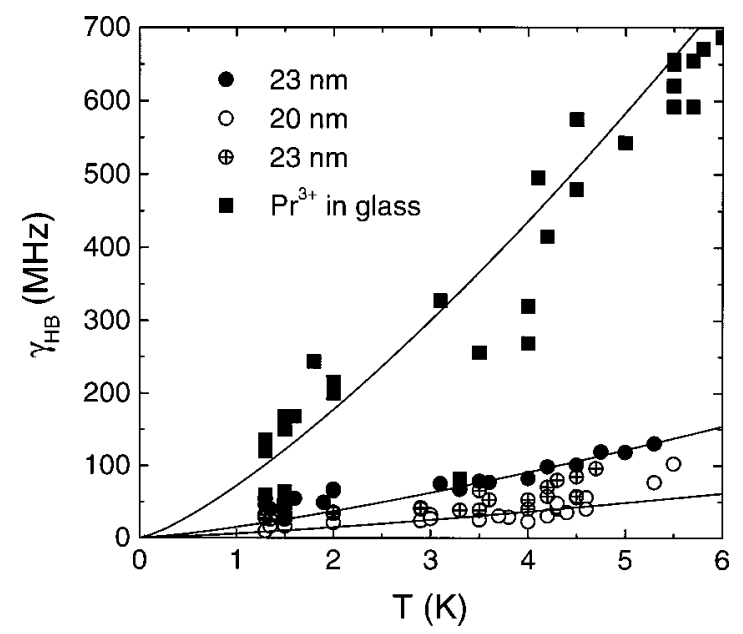

FIG. 3. Temperature dependence of the hole width for $\mathrm{Pr}^{3+}$ in $\mathrm{LaF}_{3}$ nanocrystals embedded in oxyfluoride glass ceramics and for $\mathrm{Pr}^{3+}$ directly doped into the glass matrix. Solid lines: $\gamma_{\mathrm{HB}} \sim T^{1.3}$. that the homogeneous broadening of optical transitions of $\mathrm{RE}$ ions in nanocrystals embedded in amorphous materials is determined by interactions with the TLS's of the amorphous matrix. The drastic effects on the magnitude and temperature dependence of the hole linewidth upon embedding the nanocrystals into a polymer $\left(\mathrm{Y}_{2} \mathrm{O}_{3}: \mathrm{Eu}^{3+}\right)$ and a glass $\left(\mathrm{LaF}_{3}: \mathrm{Eu}^{3+}, \mathrm{LaF}_{3}: \mathrm{Pr}^{3+}\right)$ support this suggestion and provide direct evidence for the long-range character of the TLS-RE interaction.

In order to obtain a semiquantitative description of the dependence of the homogeneous broadening on the size of the nanocrystals we apply the approach of Lyo ${ }^{1}$ and Molenkamp and Wiersma ${ }^{3}$ as restated by Huber ${ }^{5}$ and Silbey and Kassner ${ }^{4}$ to calculate the homogeneous broadening caused by the flipping of TLS's. In this approach the homogeneous broadening is calculated as a sum of contributions of each TLS. Though questioned by Huber and co-workers, 2,5 this approach gives the same results as an alternative approach ${ }^{2,5}$ for the case of dipole-dipole interactions. It has the advantage that it provides a simple means to calculate the dependence of the homogeneous broadening on the nanoparticle size since it makes it possible to exclude the nanocrystal volume about the RE ion that does not contain any TLS's. The homogeneous linewidth for an ion in the glass may be expressed, for the case of dipole-dipole interactions $\left(\sim A / r^{3}\right),{ }^{1,3,4,5}$ as

$$
\gamma_{h}=4 \pi R N_{0} \int_{0}^{r_{c}} r^{2} d r+2 \pi \frac{N_{0} A^{2}}{R} \int_{r_{c}}^{\infty} r^{-4} d r,
$$

where $N_{0}$ is the spatial density of tunneling systems, $R$ is the relaxation rate of the tunneling systems, and $r_{c}$ is a cutoff distance given by $A / r_{c}^{3}=R$, below which the TLS-ion interaction is considered distance independent. The first term describes the contribution of "near" TLS; the second accounts for interactions with more distant TLS's whose interaction with the RE ions falls according to the dipole-dipole nature of the interaction. In order to exclude the nanocrystal volume that contains no TLS, the integration should be performed excluding $r<r_{0}$ ( $r_{0}$ - the nanoparticle radius). For this semiquantitative description we consider all RE ions to be located at the center of the nanocrystals. This will result in an overestimate for $r_{c}$ since the most probable nearest distance of the RE to the nanoparticle surface is somewhat less than $r_{0}$. We also neglect the difference in elastic properties of the glass and crystal in calculating the elastic interaction. Then we have

$$
\gamma_{h}= \begin{cases}2 \pi N_{0} A-\frac{4 \pi}{3} N_{0} R r_{0}^{3} & \left(r_{0}<r_{c}\right), \\ \frac{2 \pi N_{0} A^{2}}{3 R} r_{0}^{-3} & \left(r_{0}>r_{c}\right) .\end{cases}
$$

In Fig. $4, \gamma_{n}\left(r_{0}\right)$ of Eq. (2) is plotted vs $r_{0} / r_{c}$ normalized so that $\gamma_{h}=1$ at $r_{0}=0$. On the same figure, the experimental hole widths $(T=5 \mathrm{~K})$ obtained with oxyfluoride glass ceramic samples containing $\mathrm{LaF}_{3}: \mathrm{Eu}^{3+}$ nanocrystals of different sizes (Fig. 2) are normalized to $\gamma_{h}\left(r_{0}=0\right)=1\left(\gamma_{h}=1\right.$ for 


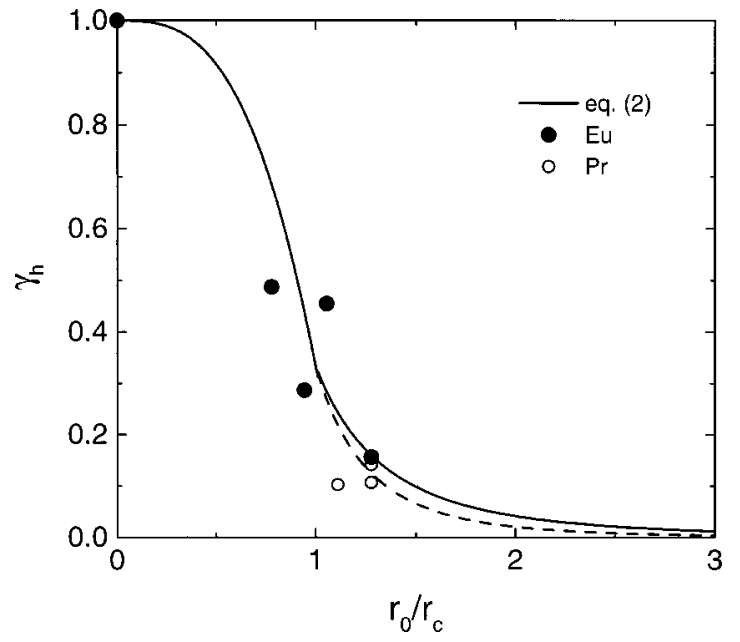

FIG. 4. The dependence of the homogeneous linewidth on nanocrystals radius (normalized to $\gamma_{h}=1$ for ions in glass). Solid line-equation (2): circles-experimental data for $\mathrm{Eu}^{3+}$ (solid) and $\mathrm{Pr}^{3+}$ (open) in $\mathrm{LaF}_{3}$ nanocrystals in oxyfluoride glass ceramics.

ions in glass) and fitted to the curve [Eq. (1)] using $r_{c}$ as an adjustable parameter. The hole widths at $T=5 \mathrm{~K}$ were estimated from the experimental data (Fig. 2) by fitting the linear $\gamma_{h}(T)$ dependence to the experimental hole widths in the vicinity of $T=5 \mathrm{~K}$. The temperature $T=5 \mathrm{~K}$ was chosen because around this temperature we have the most complete set of data for all nanoparticles sizes. At the same time the data do not suggest a significant temperature dependence of $r_{c}$. A reasonable fit of the experimental data to Eq. (2) occurs at $r_{c}=9 \mathrm{~nm}$, which may be considered as an independent estimate of $r_{c}$ for Eu doped oxyfluoride glass. The more limited set of data (obtained with the samples with different chemical compositions) for $\operatorname{Pr}^{3+}$ doped samples, also shown on Fig. 4, is also consistent with Eq. (2) assuming the same value of $r_{c}=9 \mathrm{~nm}$. The estimate of the critical length $r_{c}$ depends, mainly, on the comparison of the homogeneous broadening in the glass component $\left(r_{0} / r_{c}=0\right)$ and in the crystallites, so the small range of crystallite sizes does not significantly affect the value of $r_{c}$. The dashed curve in Fig. 4 presents the $\sim r_{0}^{-4}$ behavior of $\gamma_{h}\left(r_{0}\right)$ appropriate for the case $r_{0}>r_{c}$ (normalized) which would correspond to the situation in which the dominant role in homogeneous broadening belongs to the TLS located at the nanocrystal-glass interface if their concentration was much greater than that in the bulk. Data for larger nanocrystals are necessary to exclude (or confirm) such a possibility.

While the calculation using Eq. (2) is not very rigorous, it does provide an estimate of the dependence of the homogeneous broadening of the optical transitions of rare-earth ions on the size of nanocrystals and supports the hypothesis that the homogeneous linewidth is dominated by interactions with the TLS's of the glass matrix. A more careful theoretical treatment as well as experimental studies with nanocrystals of larger size are necessary to further test this hypothesis. Such studies are now in progress.

We thank Roger M. Macfarlane and David L. Huber for helpful discussion and the National Science Foundation, Grant No. DMR-9871864 for support of this research.
${ }^{1}$ S. K. Lyo, Phys. Rev. Lett. 48, 688 (1982).

${ }^{2}$ D. L. Huber, M. M. Broer, and B. Golding, Phys. Rev. Lett. 52, 2281 (1984); Phys. Rev. B 33, 7297 (1986).

${ }^{3}$ L. W. Molenkamp and D. A. Wiersma, J. Chem. Phys. 83, 1 (1985).

${ }^{4}$ R. Silbey and K. Kassner, J. Lumin. 36, 283 (1987).

${ }^{5}$ D. L. Huber, J. Lumin. 36, 307 (1987).

${ }^{6}$ M. J. Dejneka, MRS Bull. 23, 57 (1998); J. Non-Cryst. Solids 239, 149 (1998).

${ }^{7}$ W. R. Babbitt and T. W. Mossberg, Opt. Commun. 65, 185 (1988).

${ }^{8}$ M. Zhu, W. R. Babbitt, and C. M. Jefferson, Opt. Lett. 20, 2514 (1995).
${ }^{9}$ S. Kroll and U. Elman, Opt. Lett. 18, 1834 (1993).

${ }^{10}$ S. P. Feofilov, A. A. Kaplyanskii, R. I. Zakcharchenya, Y. Sun, K. W. Jang, and R. S. Meltzer, Phys. Rev. B 54, 3690 (1996).

${ }^{11}$ K. S. Hong, R. S. Meltzer, B. Bihari, D. K. Williams, and B. M. Tissue, J. Lumin. 76-77, 234 (1998).

${ }^{12}$ K. S. Hong, R. S. Meltzer, S. P. Feofilov, R. I. Zakharchenya, W. Jia, H. Liu, B. Tissue, and H. B. Yuan, J. Lumin. 83-84, 393 (1999).

${ }^{13}$ R. M. Macfarlane and M. J. Dejneka, Opt. Lett. 26, 429 (2001).

${ }^{14}$ B. Bihari, H. Eilers, and B. M. Tissue, J. Lumin. 75, 1 (1997).

${ }^{15}$ Th. Schmidt, R. M. Macfarlane, and S. Völker, Phys. Rev. B 50, 15707 (1994).

${ }^{16}$ R. M. Macfarlane and R. M. Shelby, J. Lumin. 36, 179 (1987). 